\title{
The affective consequences of visual attention in preview search
}

\author{
MARK J. FENSKE, JANE E. RAYMOND, and MELINA A. KUNAR \\ University of Wales, Bangor, Wales
}

\begin{abstract}
Comparisons of emotional evaluations of abstract stimuli just seen in a two-object visual search task show that prior distractors are devalued, as compared with prior targets or novel items, perhaps as a consequence of persistent attentional inhibition (Raymond, Fenske, \& Tavassoli, 2003). To further explore such attention-emotion effects, we measured search response time in a preview search task and emotional evaluations of colorful, complex images just seen therein. On preview trials, the distractors appeared 1,000 msec before the remaining items. On no-preview trials, all the items were presented simultaneously. A single distractor was then rated for its emotional tone. Previewed distractors were consistently devalued, as compared with nonpreviewed distractors, despite longer exposure and being associated with an easier task. This effect was observed only in the participants demonstrating improved search efficiency with preview, but not in others, indicating that the attentional mechanisms underlying the preview benefit have persistent affective consequences in visual search.
\end{abstract}

Selective attention and emotion are the two main brain systems used to prioritize human behavioral responses to visual stimuli. Coordinated behavior thus depends on cooperation and rapid communication between these systems. Indeed, their interaction is supported by neural connections (Amaral \& Price, 1984) and shared brain areas (Armony \& Dolan, 2002; Bush, Luu, \& Posner, 2000). Emotion-attention interactions have been evident in numerous studies reporting that the emotional salience of a stimulus can modulate the speed and efficiency of attentional processes (e.g., Eastwood, Smilek, \& Merikle, 2001; Fenske \& Eastwood, 2003; Fox, Russo, Bowles, \& Dutton, 2001), indicating that emotion can drive attention.

Recently, Raymond, Fenske, and Tavassoli (2003) demonstrated that emotion-attention interactions are not solely unidirectional; attention can also drive emotional responses. The participants in their study evaluated colorful abstract images that they had just viewed for $100 \mathrm{msec}$ in a simple two-object visual search task. Images seen previously as distractors were rated as being less cheerful than images seen previously as targets. Prior distractors were also rated as being less cheerful than items not seen before. This attention-emotion effect shows that the active attentional state during a brief exposure can modulate subsequent affective responses. Such a result is not easily explained by theories invoked to explain other preexposure effects on stimulus appraisal. With the mere exposure effect (Bornstein, 1989;

This work was supported by ESRC-ROPA Grant R022250212 to J.E.R. and by an ESRC postdoctoral fellowship to M.A.K. Address correspondence to M. J. Fenske, MGH-Martinos Center for Biomedical Imaging, Harvard Medical School, Charlestown, MA 02129 (e-mail: fenske@nmr.mgh.harvard.edu).
Zajonc, 2001), for example, passive repeated exposure to stimuli produces subsequent evaluations that are more positive than those given to similar but novel stimuli, even when the preexposed stimuli are not recognized. A widely accepted explanation for this effect is that passive, low-level perceptual encoding experience facilitates subsequent perception, causing feelings of familiarity to be interpreted as preference. The affective correlates of fluency, however, are thought to be distinctively positive (e.g., Reber, Winkielman, \& Schwarz, 1998; Winkielman \& Cacioppo, 2001), which cannot explain why distractors were devalued, in the Raymond et al. studies, relative both to targets seen for the same amount of time and to novel stimuli. Another explanation for the mere exposure effect is that an enhanced positive affect results from the association of the passively viewed stimuli in the exposure phase with a lack of unpleasant events (Zajonc, 2001). This account also fails to explain the devaluation involved in the attention-emotion effect, because ratings from both prior targets and prior distractors were obtained only after successful visual search trials (i.e., those with no aversive consequences).

To explain their results, Raymond et al. (2003) proposed that attentional inhibition directed at stimuli during visual search may serve to reduce the emotional salience of distracting stimuli so that, in the future, they will be less likely to attract attention. Accordingly, prior inhibition leads to item devaluation when previously distracting stimuli are presented in an evaluation task. The purpose of the present experiment was to test the generality of the attention-emotion effect, using a more complex visual search task that was temporally segmented to produce a phenomenon known as the preview effect (Watson \& Humphreys, 1997, 2000). The preview effect consists of substantially improved efficiency in visual 
search when a subset of distractors is presented for about $1 \mathrm{sec}$ before the remaining items in the search array. This task was chosen because one prevalent account of the preview effect is that top-down inhibition is applied to previewed distractors (e.g., Watson \& Humphreys, 1997, 2000). If this is the case and inhibition causes affective devaluation of stimuli, a search task that produces a preview benefit should also produce a subsequent devaluation of previewed distractors. In the present experiment, this possibility was examined.

In a preview search paradigm, the target is defined by a unique two-feature conjunction (here, color and element shape), with each distractor sharing one of these features. Critically, the search display is temporally segregated on half of the trials. On these preview trials, a subset of distractor items (the preview set) that share the same target feature (here, element shape) are presented $1,000 \mathrm{msec}$ before the remaining distractors and the target. On no-preview trials, the preview set is presented simultaneously with the rest of the items, making the task a difficult conjunction search task. The remaining distractors (the search set) also share a single feature with the target (here, color) and are always presented simultaneously with the target. If preview set distractors can be fully ignored on preview trials, search set distractors create an easy feature search task. A preview benefit is said to be present when search is more efficient (i.e., when search times plotted as a function of set size produce a flatter slope) in the preview condition than in the no-preview condition.

Indeed, Watson and Humphreys (1997) showed that search in the preview condition can be as efficient as when the preview set is entirely absent. To account for this and related findings, they posited a visual-marking process that involves top-down inhibitory filtering of previewed items to prevent them from interfering with the subsequent search task (e.g., Watson \& Humphreys, 2000). Regarding the subsequent evaluation of previewed versus not-previewed distractors, this top-down inhibition account of the preview effect, in light of Raymond et al.'s (2003) evidence that inhibited items are devalued, predicts that previewed distractors should be devalued relative to nonpreviewed items.

In the present study, we utilized a two-feature conjunction search task in a modified preview-search setup and followed each visual search trial with a rating task involving one item from the preceding search array. The stimuli were colorful abstract patterns (Figure 1) chosen to engage the interest of the participants. In the preview condition, all the preview set patterns (green squares) were presented for $1,000 \mathrm{msec}$ prior to the search set patterns (red circles) and the target (red squares). In the nopreview condition, all the items (preview set, search set, and target) were presented simultaneously. The number of distractors was varied so that search efficiency measured in the preview condition could be compared with that measured in the no-preview condition. After each search trial, a single distractor (from the preview set or the search set) was presented in the same location as it originally appeared in the search task, and the participants were asked to rate their emotional response to it (i.e., how cheerful or dreary it appeared). Together, these two tasks enabled us to identify those individuals showing a preview benefit in the search task and then compare their ratings for previewed versus not-previewed distractors.

\section{METHOD}

\section{Participants}

Forty-nine University of Wales, Bangor students (mean age, 24.1 years; 24 females), naive as to the aims of the experiment, participated in exchange for course credit. Twenty-five participants rated images for cheerfulness; 24 others rated images for dreariness. The participants gave informed consent prior to participation.

\section{Apparatus and Stimuli}

The experiment was conducted on a Pentium III computer with a 33-cm color monitor $(100 \mathrm{~Hz}, 1,024 \times 768$ resolution) running EPrime 1.1 software (Schneider, Eschman, \& Zuccolotto, 2002). The stimuli appeared on a uniform white field at a 70 -cm average viewing distance. Alphanumeric stimuli (i.e., "+” and "?") appeared in black 18-point Courier-New font, except where stated.

Each item in the search array was a $1-\mathrm{cm}\left(0.82^{\circ}\right)$ square pattern composed of 32 same-shaped elements (squares or circles). In each pattern, the elements were mostly (24/32) red (RGB: $255,0,0)$ or mostly green (RGB: $0,255,0)$. The remaining elements had randomly selected hues (8-bit palette). Element size (diameter or side length) was selected randomly from two ranges: large $(1.5-2.5 \mathrm{~mm})$ or small $(0.5-1.0 \mathrm{~mm})$. Red squares were the predominant elements for targets and were mostly (24/32) either large or small. Distractor patterns were composed of equal proportions of large and small elements. Preview set distractor elements were square and mostly green, whereas search set distractor elements were circular and mostly red. Each pattern could appear at 1 of 16 possible locations within a $4.91^{\circ}$ square. Item location was confined to regular grid locations, with $1.23^{\circ}$ between the centers of each potential location.

\section{Procedure and Design}

The general procedure and the sequence of trial events for each condition are shown in Figure 1B. At the start of each trial, a fixation cross was presented centrally for $500 \mathrm{msec}$ and remained visible throughout the trial. In the preview condition, the preview set was then displayed for 1,000 msec. The target and search set distractors were then added, and all the items remained visible until a response was made. In the no-preview condition, the fixation cross increased from 18 to 32 points after its initial appearance to provide a temporal signal equal to that provided by the preview display in the preview condition. The larger fixation cross was viewed for $1,000 \mathrm{msec}$, and then the search set, the preview set, and the target appeared simultaneously, remaining visible until a response. In both conditions, the task was to indicate the size of the predominant target element (large or small) by pressing one of two keys as quickly as possible. After this, a 150-msec blank interval preceded a central question mark prompt, lasting 1,000 msec. After another 150-msec blank interval, a single distractor from the immediately preceding search task appeared at the same location as that at which it had originally appeared for $350 \mathrm{msec} .{ }^{1}$ On half of the trials, this was a search set distractor, and on the remaining trials, it was a preview set distractor. The participants rated each item's emotional tone, using the number keys 1 through 5. Half rated the images for cheer- 
A. Targets

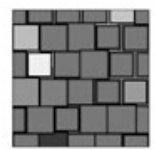

Large squares

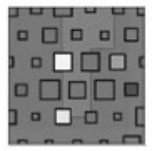

Small squares
Distractors

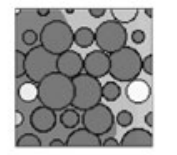

Search set Preview set

\section{B. Preview condition}

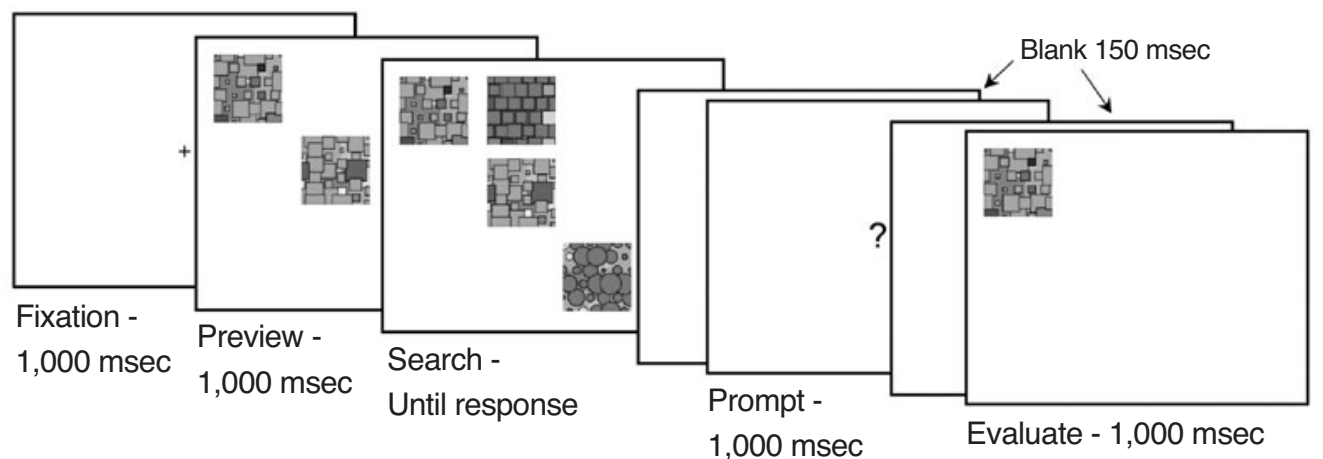

No-preview condition

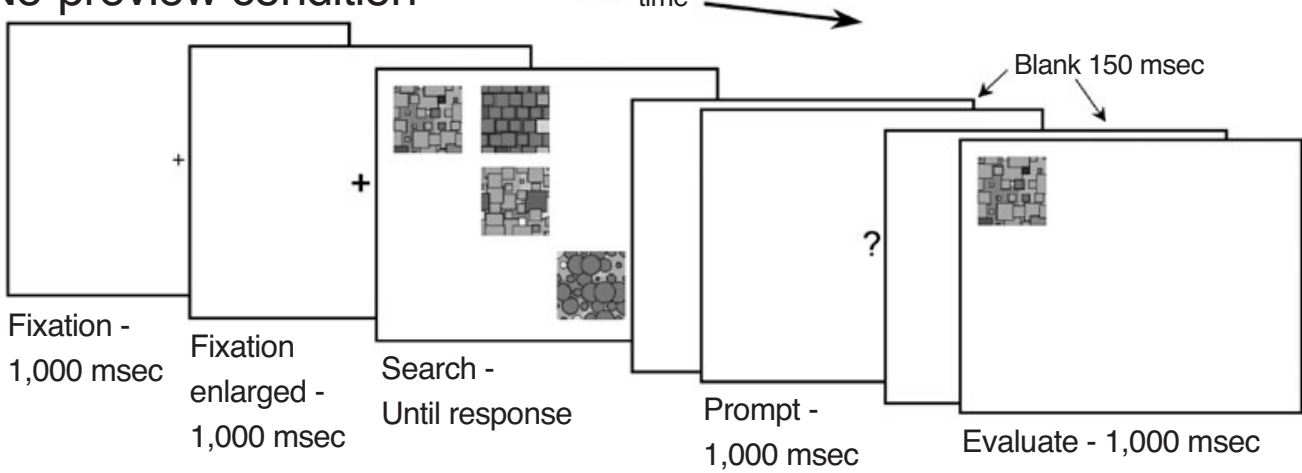

Figure 1. (A) Types of stimuli used. (B) Sequence of events for experimental trials in the preview (top) and the no-preview (bottom) conditions. Both conditions required first a visual search response for a target made from red squares and then an evaluation of the cheerfulness or dreariness of a single distractor item made from green squares or red circles from the preceding array. For the no-preview condition, the fixation cross was enlarged for 1,000 msec, whereas in the preview condition, the preview set distractors were presented at the corresponding point in time and for the same duration.

fulness, and the remaining participants used the same keys to rate the images for dreariness. For both groups, the response range was anchored by the same labels for the lowest $(1$, not at all $)$, the middle (3, maybe), and the highest (5, definitely) values.

For search, set size varied among 4, 8, and 16 items, meaning that the number of distractors was always 3,7 , or $15(2,4$, or 8 for the preview set, and 1, 3, or 7 for the search set, respectively). Every distractor was a unique exemplar. The target contained mostly small squares on half the trials and mostly large squares on the remaining trials. The locations of the target and the distractors were randomly assigned.

An experimental block (120 trials) containing an equal number of trials from each condition formed by the factorial combination of search condition (preview or no preview), set size $(4,8$, or 12), target type (small or large squares), and to-be-rated item type (preview set or search set) was preceded by a similarly constructed set of 12 practice trials. Trial order was randomly determined. The experiment lasted approximately $30 \mathrm{~min}$.

\section{Data Analysis and Response Scale Conversion}

Only scores for trials in which target square size was correctly identified were analyzed further. This criterion excluded only $3.9 \%$ of the trials; accuracy was consistently high across response scales, conditions, set sizes, and target types. A further $2.6 \%$ of the remaining trials were removed using a recursive procedure (Van Selst \& Jolicœur, 1994) to identify reaction time (RT) outliers from each cell. The rating responses from the remaining trials were converted into numerical values reflecting the same emotional tone for each response scale, with smaller values reflecting more negative evaluations. Specifically, responses using the cheerful scale were assigned a value of 1 for not at all cheerful and 5 for definitely cheerful. Conversely, responses using the dreary scale were assigned 1 for definitely dreary and 5 for not at all dreary. If the observers expressed similar emotional responses, despite using oppositely valenced response scales, there should be no difference between the groups in their converted rating scores. All further references to rating values refer to these converted values. 


\section{RESULTS}

\section{Visual Search RTs}

Mean correct search RTs and search slopes relating mean RTs across increasing set size, for each condition, were calculated for each participant. These data were then assessed using two simple criteria derived from the extant preview search literature to ascertain whether a preview benefit occurred for each individual. The participants who showed evidence of a preview benefit were placed in the preview benefit (PB) group, whereas those who showed no evidence of a preview benefit were placed in the no-preview benefit (no-PB) group. The criteria for PB group membership stipulated that a participant's search slope must be shallower for the preview condition than for the no-preview condition (e.g., Watson \& Humphreys, 1997) or the overall mean RT had to be shorter in the preview condition than in the no-preview condition (Jiang, Chun, \& Marks, 2002). Twenty-nine participants met one or both criteria (PB group), and 20 failed to meet either (no-PB group). To ensure that group differences were not due to discrepancies in search trial accuracy, a 2 (group: PB vs. no-PB) $\times 2$ (search condition: preview vs. no preview) $\times 3$ (set size) mixed-factors analysis of variance (ANOVA) was conducted on the proportions of search trial errors. This analysis revealed no systematic effects of set size or search condition and no difference in the overall search error rates for the $\mathrm{PB}$ $(3.9 \%)$ and the no-PB $(4.5 \%)$ groups (all $F \mathrm{~s}<1)$.
Mean correct search RTs are shown separately for each group, as a function of set size and search condition, in Figure 2. A 2 (search condition: preview vs. no preview) $\times 3$ (set size) repeated measures ANOVA was conducted on correct search RTs separately for each group, the results of which are shown in Table 1, along with other critical results of the RT analysis (also displayed in Figure 2). The critical benefit of preview search is clearly present for the $\mathrm{PB}$ group and absent for the noPB group. Indeed, whereas the PB group produced a significantly steeper search slope in the no-preview condition than in the preview condition, the no-PB group produced a marginally shallower search slope in the nopreview condition than in the preview condition.

\section{Response Scale Valence}

We first analyzed all the ratings to assess the effect of the response scale valence. For both the PB (20 cheery, 9 dreary) and the no-PB ( 6 cheery, 14 dreary) groups, a mixed-factors ANOVA, using response scale (cheerful vs. dreary) as a between factor and search condition (preview vs. no preview), set size (4 vs. 8 vs. 16), and distractor type (preview set vs. search set) as within factors, revealed that neither the main effect of scale valence nor any of its higher order interactions was significant (all $\left.F_{\mathrm{s}}<1, p>.10\right)$. This finding converges with our previous work (Raymond et al., 2003) in which these scales were used to suggest that ratings reflect judgments about the perceived emotional tone of items, rather than a sim-

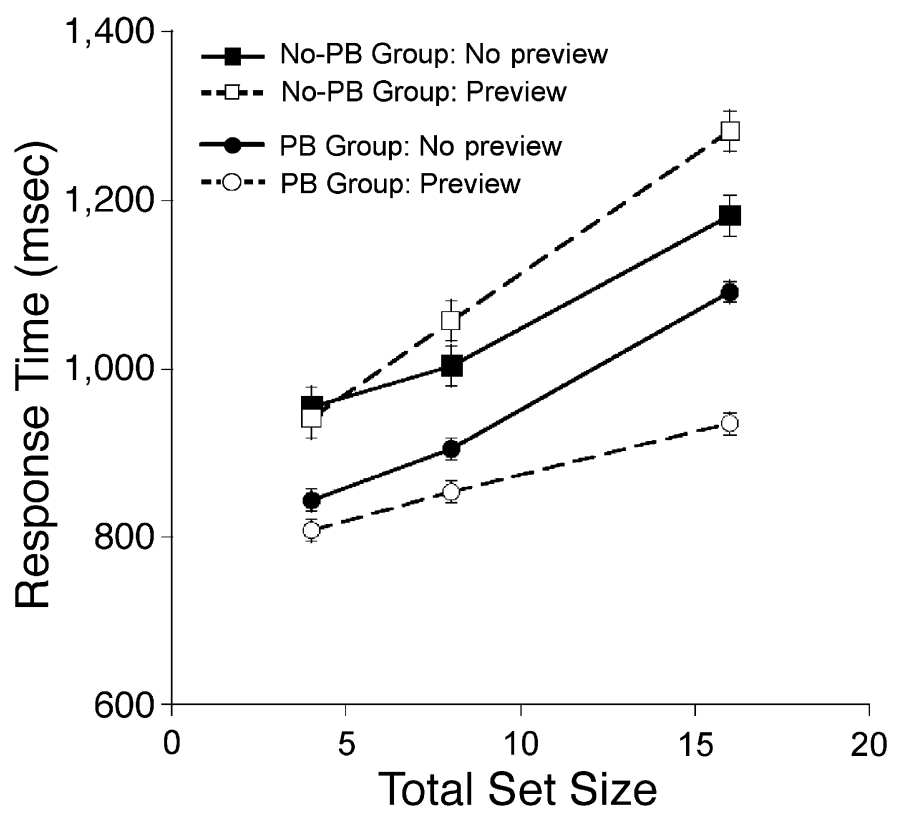

Figure 2. Mean search response times for preview and no-preview conditions as a function of total set size, plotted separately for the preview benefit (PB; $n=29)$ and the no-preview benefit (no-PB; $n=20)$ groups. Error bars represent standard errors based on the procedure suggested by Loftus and Masson (1994) for within-subjects designs. 
Table 1

Correct Search Trial Response Time (RT) Data and Results of a 2 (Search Condition) $\times 3$ (Set Size) Repeated Measures ANOVA for Participants Meeting (PB Group) and Not Meeting (No-PB Group) the Basic Criteria for Preview Benefit in Visual Search

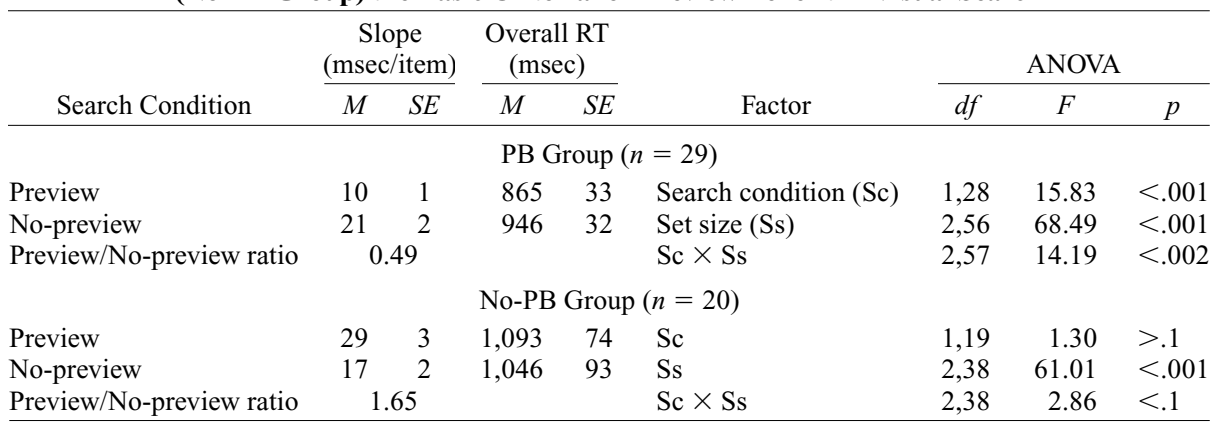

Table 2

Mean Rating Responses and Standard Errors of the Means for Each Condition (Averaged Across Set Size) for the Preview Benefit (PB) and the No-Preview Benefit (No-PB) Groups

\begin{tabular}{|c|c|c|c|c|c|c|c|c|}
\hline \multirow[b]{3}{*}{ Group } & \multicolumn{4}{|c|}{ Preview Set } & \multicolumn{4}{|c|}{ Search Set } \\
\hline & \multicolumn{2}{|c|}{ Preview } & \multicolumn{2}{|c|}{ No Preview } & \multicolumn{2}{|c|}{ Preview } & \multicolumn{2}{|c|}{ No Preview } \\
\hline & $M$ & $S E$ & $M$ & $S E$ & $M$ & $S E$ & $M$ & $S E$ \\
\hline PB group $(n=29)$ & 2.69 & 0.04 & 2.77 & 0.04 & 3.62 & 0.04 & 3.56 & 0.04 \\
\hline No-PB group $(n=20)$ & 2.96 & 0.06 & 2.93 & 0.06 & 3.02 & 0.06 & 2.96 & 0.06 \\
\hline
\end{tabular}

ple motor response bias. Accordingly, the data for all the subsequent analyses were pooled across this factor.

\section{Emotional Response Data}

The mean ratings for each condition are shown in Table 2, and the mean rating differences (preview minus no-preview condition) are plotted for each distractor type for each group in Figure 3. For the PB group, preview had a devaluating effect on preview set distractors, reducing the no-preview mean rating of 2.77 to the preview mean rating of 2.69. In contrast, preview acted to slightly elevate ratings for search set items from a mean of 3.56 without preview to 3.62 with preview. A 2 ( search condition) $\times 3$ (set size) $\times 2$ (distractor type) repeated measures ANOVA revealed that this search condition $\times$ distractor type interaction was indeed significant $[F(1,28)=4.82, p<.05]$, confirming that the effect of preview on emotional responses was qualitatively different for preview set versus search set distractors. Importantly, whereas a 2 (search condition) $\times 3$ (set size) repeated measures ANOVA on the ratings for preview set distractors revealed a significant effect of search condition $[F(1,28)=4.26, p<.05]$, this effect was nonsignificant in a similar analysis of search set distractor ratings $[F(1,28)=2.25, p>.1]$. Neither the main effects of set size nor the set size $\times$ search condition interactions were significant in the analysis of the ratings given for either preview set distractors $[F(1,19)<1, p>$ $.10]$ or search set distractors $[F(1,19)=1.63, p>.10]$.
In contrast to the rating results for the PB group, a similar set of analyses conducted on the ratings provided by the no-PB group revealed that neither the main effects of search condition or set size nor any of the higher order interactions were significant for either type of distractor ( $p>.10$ for each). The lack of any appreciable effect of preview on the ratings obtained from the no-PB group can be seen in Figure 3.

\section{DISCUSSION}

Using a preview search paradigm (Watson \& Humphreys, 1997), we created a visual search task involving complex stimuli that required a conjunction of color and internal element shape when they were presented simultaneously for target detection. On half of the trials, all the distractors in the nontarget color were previewed for $1,000 \mathrm{msec}$. The visual-marking hypothesis (e.g., Watson \& Humphreys, 1997, 2000) proposes that all previewed items in this situation are inhibited via a top-down selective attention mechanism that allows target search in the remaining items to become a simple feature discrimination task. Accordingly, effective visual marking of previewed items produces improved search efficiency, reflected by a shallower slope in the function relating search RT and set size. This preview benefit was observed in $59 \%$ of the participants in this study. The purpose of our experiment was to determine whether the hypothesized inhibition of previewed distractors would also cause 


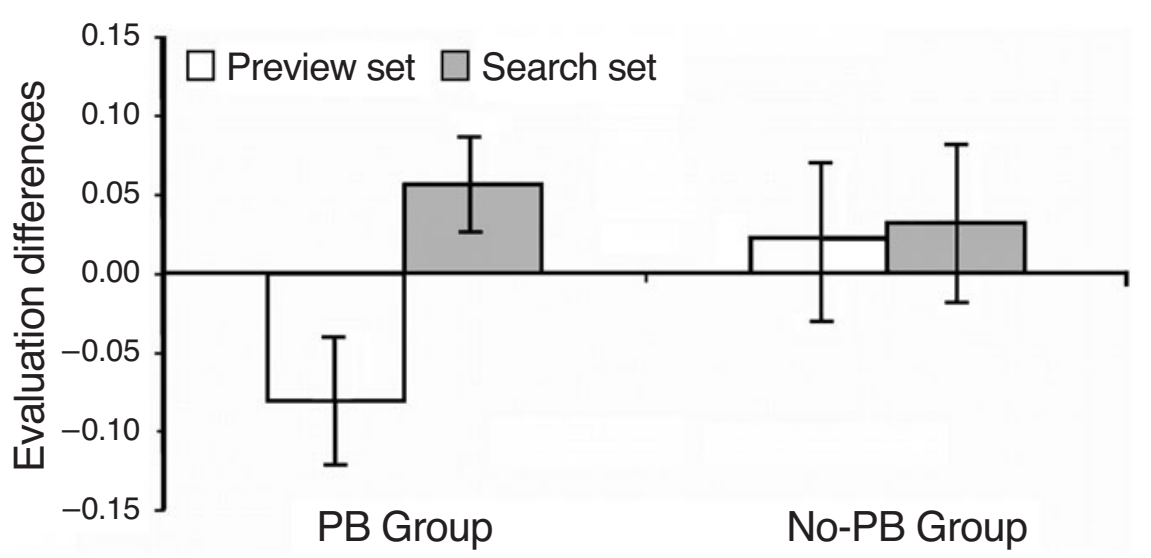

\begin{abstract}
Figure 3. Mean difference in evaluation of distractors in the preview versus the no-preview conditions for preview set and search set distractors, plotted separately for the preview benefit $(\mathrm{PB} ; n=29)$ and the no-preview benefit (no-PB; $n=20)$ groups. Negative numbers on the $y$-axis reflect an affective devaluation of the items seen in the preview condition. Error bars represent standard errors based on the procedure suggested by Loftus and Masson (1994) for within-subjects designs.
\end{abstract}

a subsequent emotional devaluation of these items, since previous studies of attention-emotion effects have suggested that selective attentional inhibition leads to devaluation (Raymond et al., 2003). Our results are clear. For the participants showing a preview benefit, the previewed distractors were significantly devalued, as compared with similar distractors not seen in preview. In contrast, the individuals that did not show a preview benefit also failed to show a devaluation of previewed distractors.

In the original demonstration of the attention-emotion effect, Raymond et al. (2003) found that participants rated items just seen as distractors in a simple two-item search task less positively than those just seen as targets and less positively than novel baseline items. Because this effect is not easily explained by perceptual fluency differences in distractors versus targets (Reber et al., 1998) or by conditioned associations with nonaversive events (Zajonc, 2001), they proposed that attentional inhibition directed at stimuli during visual search may serve to reduce the emotional salience of distracting stimuli. If the inhibition account of the preview effect is correct, the present results provide converging support for Raymond et al.'s inhibition account of the effect of selective attention on emotional evaluation. In contrast, these results do not support perceptual fluency or conditioning explanations for preexposure effects on evaluative responses. In the present experiment, distractors from the preview set were viewed for one full second longer in the preview condition than they were in the nopreview condition. A perceptual fluency account predicts that affective appraisal of these stimuli should be more positive with preview than without, but this was clearly not the case. Furthermore, preview trials were easier than no-preview trials, as evidenced by generally shorter RTs in the former condition (in the PB group).
Conditioning explanations of preexposure effects on affective appraisal predict that images associated with these trials should be evaluated more positively. The present finding does not support this prediction either. Indeed, only the participants who clearly showed a preview benefit in the preceding search tasks also showed clear, qualitatively different effects of preview on the emotional ratings of previewed versus nonpreviewed distractors. This result indicates that the attentional state used to process novel stimuli determines how they will be emotionally evaluated subsequently, thereby replicating the essential finding in Raymond et al. and demonstrating its generality with a more complex visual search task.

We chose to compare affective evaluations of distractors in preview search because attentional inhibition may cause affective devaluation of stimuli (Raymond et al., 2003) and because a prevalent account of the preview effect is that top-down inhibition is applied to previewed distractors (e.g., Watson \& Humphreys, 1997, 2000). However, other explanations of preview benefit also merit consideration. Donk and Theeuwes (2001), for instance, argued against an inhibition account, suggesting instead that preview benefit occurs because search set items capture attention when they onset, giving them priority access to processing over preview set items in the preview condition. Accordingly, previewed distractors may be liked less than nonpreviewed distractors because of insufficient processing when attention is captured away by search set items. Differences in attentional capture by stimulus onset cannot, however, account for our previous finding that distractors are devalued when presented simultaneously with targets (Raymond et al., 2003). Another account of the preview effect is that it occurs as a result of perceptual grouping based on temporal segregation of preview versus search set items 
(Jiang, Marks, \& Chun, 2002). Although more positive ratings for nonpreviewed versus previewed distractors is compatible with this view, it cannot account for the distractor devaluation in Raymond et al.'s study, in which stimuli were simultaneously presented. In addition, both groups in the present experiment saw the same stimuli and were, presumably, susceptible to the same bottom-up perceptual influences, but only the PB group showed a devaluation of the previewed items. Thus, although the present experiment provides no direct evidence regarding current accounts of preview effects, it may nonetheless be parsimonious to explain the devaluation of previewed items through a common attentional mechanism that facilitates visual search by selectively inhibiting previewed items (visual marking), which thereby reduces their emotional salience.

Note that we use the term devaluation to indicate simply that stimuli seen in some situations are valued less than similar stimuli seen in other situations. We do not necessarily mean to imply that devalued stimuli have been rendered affectively negative (e.g., unpleasant or threatening). Rather, it seems more likely that if attentional inhibition is what acts to reduce evaluative ratings, devaluation in the current context may reflect a process of affective neutralization (i.e., a reduction in the emotional salience of previously inhibited stimuli). An attentionally driven process that reduces the emotional salience of distracting stimuli makes sense because it would serve to reduce the capacity of such stimuli to capture attention in future encounters.

\section{REFERENCES}

Amaral, D. G., \& Price J. L. (1984). Amygdalo-cortical projections in the monkey (Macaca fascicularis). Journal of Comparative Neurology, 230, 465-496.

ARMONY, J. L., \& DolAN, R. J. (2002). Modulation of spatial attention by fear conditioned stimuli: An event-related fMRI study. Neuropsychologia, 40,817-826.

BornsteIn, R. F. (1989). Exposure and affect: Overview and metaanalysis of research, 1968-1987. Psychological Bulletin, 106, 265289.

Bush, G., LuU, P., \& Posner, M. I. (2000). Cognitive and emotional influences in anterior cingulate cortex. Trends in Cognitive Sciences, $\mathbf{4}_{2}$ 215-222.

Donk, M., \& TheEuwes, J. (2001). Visual marking beside the mark:
Prioritizing selection by abrupt onsets. Perception \& Psychophysics, 63, 891-900.

EasTwood, J. D., SMilek, D., \& MeriKle, P. M. (2001). Differential attentional guidance by unattended faces expressing positive and negative emotion. Perception \& Psychophysics, 63, 1004-1013.

Fenske, M. J., \& EAstwood, J. D. (2003). Modulation of focused attention by faces expressing emotion: Evidence from flanker tasks. Emotion, 3, 327-343.

Fox, E., Russo, R., Bowles, R., \& Dutton, K. (2001). Do threatening stimuli draw or hold visual attention in subclinical anxiety? Journal of Experimental Psychology: General, 130, 681-700.

JiAng, Y., Chun, M. M., \& Marks, L. E. (2002). Visual marking: Dissociating effects of new and old set size. Journal of Experimental Psychology: Learning, Memory, \& Cognition, 28, 293-302.

JiANG, Y., Marks, L. E., \& Chun, M. M. (2002). Visual marking: Selective attention to asynchronous temporal groups. Journal of Experimental Psychology: Human Perception \& Performance, 28, 717750.

LofTus, G. R., \& MAsson, M. E. J. (1994). Using confidence intervals in within-subject designs. Psychonomic Bulletin \& Review, 1, 476-490.

RaYMond, J. E., Fenske, M. J., \& TAVAssoli, N. T. (2003). Selective attention determines emotional responses to novel visual stimuli. Psychological Science, 14, 537-542.

REBER, R., WinkielmaN, P., \& SchWARZ, N. (1998). Effects of perceptual fluency on affective judgments. Psychological Science, 9, 45-48.

Schneider, W., Eschman, A., \& Zuccolotto, A. (2002). user's guide. Pittsburgh: Psychology Software Tools.

VAN SELST, M., \& JolicceUR, P. (1994). A solution to the effect of sample size on outlier elimination. Quarterly Journal of Experimental Psychology, 47A, 631-650.

Watson, D. G., \& Humphreys, G. W. (1997). Visual marking: Prioritizing selection for new objects by top-down attentional inhibition of old objects. Psychological Review, 104, 90-122.

Watson, D. G., \& HumphreYs, G. W. (2000). Visual marking: Evidence for inhibition using a probe-dot detection paradigm. Perception \& Psychophysics, 62, 471-481.

Winkielman, P., \& CACIOPPO, J. T. (2001). Mind at ease puts a smile on the face: Psychophysiological evidence that processing facilitation increases positive affect. Journal of Personality \& Social Psychology, 81, 989-1000.

ZAJONC, R. B. (2001). Mere exposure: A gateway to the subliminal. Current Directions in Psychological Science, 10, 224-228.

\section{NOTE}

1. Because visual-marking accounts suggest that the locations of previewed items may be inhibited, the locations of to-be-rated items were the same as when they were presented in the search task, in order to maximize the inhibitory effects on ratings by capitalizing on locationbased, as well as any possible nonspatial, inhibition.

(Manuscript received August 1, 2003; revision accepted for publication February 12, 2004.) 